\title{
A Characterization for Fourier Hyperfunctions
}

\author{
By \\ Jaeyoung CHUNG*, Soon-Yeong CHUNG** and Dohan $\mathrm{KIM}^{* * *}$
}

\begin{abstract}
The space of test functions for Fourier hyperfunctions is characterized by two conditions $\sup |\varphi(x)| \exp k|x|<\infty$ and $\sup |\hat{\varphi}(\xi) ! \exp h| \xi \mid<\infty$ for some $h, k>0$. Combining this result and the new characterization of Schwartz space in [1] we can easily compare two important spaces $\widetilde{F}$ and $\mathcal{S}$ which are both invariant under Fourier transformations.
\end{abstract}

\section{$\S 0$. Introduction}

The purpose of this paper is to give new characterization of the space $\mathscr{I}$ of test functions for the Fourier hyperfunctions.

In [6], K. W. Kim, S. Y. Chung and D. Kim introduce the real version of the space $\mathscr{F}$ of test functions for the Fourier hyperfunctions as follows,

$$
\mathscr{F}=\left\{\varphi \in C^{\infty} \mid \sup _{a, x} \frac{\left|\partial^{a} \varphi(x)\right| \exp k|x|}{h^{|\alpha|} \alpha !}<\infty \quad \text { for some } k, h>0\right\} .
$$

They also show the equivalence of the above definition and Sato-Kawai's original definition in complex form.

Also, in [1] J. Chung, S. Y. Chung and D. Kim give new characterization of the Schwartz space $\mathcal{S}$, i.e., show that for $\varphi \in C^{\infty}$ the following are equivalent:

(1) $\varphi \in \mathcal{S}$;

(2) $\sup \left|x^{\alpha} \varphi(x)\right|<\infty, \sup \left|\partial^{\beta} \varphi(x)\right|<\infty$ for all multi-indices $\alpha$ and $\beta$;

(3) $\sup \left|x^{\alpha} \varphi(x)\right|<\infty, \sup \left|\xi^{\beta} \hat{\varphi}(\xi)\right|<\infty$ for all multi-indices $\alpha$ and $\beta$.

In a similar fashion as above we will give new characterization of the space IF of test functions for the Fourier hyperfunctions as the main theorem in this paper which says that for $\varphi \in C^{\infty}$ the following are equivalent:

(1) $\varphi \in \mathscr{T}$;

(2) $\sup |\varphi(x)| \exp k|x|<\infty, \sup |\hat{\varphi}(\xi)| \exp h|\xi|<\infty$ for some $h, k>0$.

Observing the above growth conditions we can easily see that the space $\mathscr{T}$ which is invariant under the Fourier transformation is much smaller than

Communicated by T. Kawai, April 5, 1993. Revised May 20, 1993.

1991 Mathematics Subject Classification: 46F12, 46F15.

* Department of Mathematics, Kunsan National University, Kunsan 573-360, Korea.

** Department of Mathematics, Duksung Women's University, Seoul 132-714, Korea.

*** Department of Mathematics, Seoul National University, Seoul 151-742, Korea.

Partially supported by the Ministry of Education and GARC. 
Schwartz space $\mathcal{S}$. Since an element in the strong dual $\mathscr{I}^{\prime}$ of the space $\mathscr{T}$ is called a Fourier hyperfunction, the space $\mathscr{F}^{\prime}$ of Fourier hyperfunctions which is also invariant under the Fourier transformation is much bigger than the space $\mathcal{S}^{\prime}$ of tempered distributions.

Section 1 is devoted to providing the necessary definitions and preliminaries. We prove the main theorem in Section 2.

\section{$\S 1$. Preliminaries}

We use the multi-index notations; for $x=\left(x_{1}, \cdots, x_{n}\right), \xi=\left(\xi_{1}, \cdots, \xi_{n}\right) \in \boldsymbol{R}^{n}$ and a multi-index $\alpha=\left(\alpha_{1}, \alpha_{2}, \cdots, \alpha_{n}\right) \in \boldsymbol{N}_{0}^{n}, \partial^{\alpha}=\partial_{1}^{\alpha_{1}} \cdots \partial_{n}^{\alpha_{n}},|\alpha|=\alpha_{1}+\cdots+\alpha_{n}$ with $\partial_{\jmath}=\partial / \partial x_{\jmath}$, and $N_{0}$ the set of non-negative integers.

For $f \in L^{1}\left(\boldsymbol{R}^{n}\right)$ the Fourier transform $\hat{f}$ is the bounded continuous function in $\boldsymbol{R}^{n}$ defined by

$$
\hat{f}(\xi)=\int e^{-i x \cdot \xi} f(x) d x, \quad \xi \in \boldsymbol{R}^{n}
$$

Definition 1.1. We denote by $\mathcal{S}$ or $\mathcal{S}\left(\boldsymbol{R}^{n}\right)$ the Schwartz space of all $\varphi \in$ $C^{\infty}\left(\boldsymbol{R}^{n}\right)$ such that

$$
\sup _{x}\left|x^{\alpha} \partial^{\beta} \varphi(x)\right|<\infty
$$

for all multi-indices $\alpha$ and $\beta$.

We need the following characterization to compare the space $\mathscr{F}$ of test functions for the Fourier hyperfunctions with the above space.

Theorem 1.2 [1]. (i) The Schwartz space $\mathcal{S}$ consists of all $\varphi \in C^{\infty}\left(\boldsymbol{R}^{n}\right)$ satisfying the conditions

$$
\begin{aligned}
& \sup _{x}\left|x^{\alpha} \varphi(x)\right|<\infty, \\
& \sup _{x}\left|\partial^{\beta} \varphi(x)\right|<\infty
\end{aligned}
$$

for all multi-indices $\alpha$ and $\beta$.

(ii) Also, the Schwartz space can be characterized by the following two conditions

$$
\begin{aligned}
& \sup \left|x^{\alpha} \varphi(x)\right|<\infty, \\
& \sup \left|\xi^{\beta} \hat{\varphi}(\xi)\right|<\infty
\end{aligned}
$$

for all multi-indices $\alpha$ and $\beta$.

Now, we are going to introduce the original complex version and new real definition of test functions for the Fourier hyperfunctions as in [6], and state their equivalence. 
Definition 1.3 [6]. A real valued function $\varphi$ is in $\mathscr{F}$ if $\varphi \in C^{\infty}\left(\boldsymbol{R}^{n}\right)$ and if there are positive constants $h$ and $k$ such that

$$
|\varphi|_{k, h}=\sup _{a, x} \frac{\left|\partial^{\alpha} \varphi(x)\right|}{h^{|a|} \alpha !} \exp k|x|<\infty .
$$

Definition 1.4 [5]. A complex valued function $\varphi(z)$ is in $\mathscr{Q}_{*}$ if $\varphi(z)$ is holomorphic in a tubular neighborhood $\boldsymbol{R}^{n}+i\{|y| \leqq r\}$, for some $r$, of $\boldsymbol{R}^{n}$ and if for some $k>0$

$$
\sup _{z \in R_{n}+i\{1 y \mid \leqslant r\}}|\varphi(z)| \exp k|z|<\infty .
$$

Theorem $1.5[6]$. The space $\mathscr{F}$ is isomorphic to the space $\mathscr{P}_{*}$.

Definition 1.6. We denote by $\mathscr{F}^{\prime}$ the strong dual space of $\mathscr{I}$ and call its elements Fourier hyperfunctions.

Thus the global theory of the Fourier hyperfunctions is nothing but the duality theory for the space $\mathscr{F}$.

\section{$\S 2$. Main Theorem}

Now we shall give new characterization of the space $I$ of test functions for the Fourier hyperfunctions which is the main result in this paper.

First, we prove

Theorem 2.1. The following conditions for $\varphi \in C^{\infty}$ are equivalent:

(i) There are positive constants $k$ and $h$ such that

$$
\sup _{\alpha, x} \frac{\left|\partial^{\alpha} \varphi(x)\right| \exp k|x|}{h^{|\alpha|} \alpha !}<\infty .
$$

(ii) There are positive constants $C, k$ and $h$ such that

$$
\begin{aligned}
& \sup _{x}|\varphi(x)| \exp k|x|<\infty, \\
& \sup _{x}\left|\partial^{\alpha} \varphi(x)\right| \leqq C h^{|\alpha|} \alpha ! .
\end{aligned}
$$

(iii) There are positive constants $k$ and $h$ such that

$$
\begin{aligned}
& \sup _{x}|\varphi(x)| \exp k|x|<\infty, \\
& \sup _{\xi}|\hat{\varphi}(\xi)| \exp h|\xi|<\infty .
\end{aligned}
$$

Proof. The implications (i) $\Rightarrow$ (ii), (i) $\Rightarrow$ (iii) are trivial. So it suffices to prove the implications (iii) $\Rightarrow$ (ii) and (ii) $\Rightarrow$ (i) in order.

(iii) $\Rightarrow$ (ii): By the inequality (2.5) we have 


$$
\begin{aligned}
\left|\partial^{\alpha} \varphi(x)\right| & \leqq \frac{1}{(2 \pi)^{n}} \int\left|\xi^{a}\right||\hat{\varphi}(\xi)| d \xi \\
& \leqq \frac{M}{(2 \pi)^{n}} \int|\xi|^{\mid \alpha_{1}} \exp (-h|\xi|) d \xi \\
& \leqq \frac{M}{(2 \pi)^{n}} \sup _{\xi} \frac{|\xi|^{\mid \alpha,}}{\exp (h|\xi| / 2)} \int \exp (-h|\xi| / 2) d \xi \\
& \leqq C A^{|\alpha|} \alpha !
\end{aligned}
$$

for some positive constants $M, A$ and $C$. Thus, we obtain the condition (2.3) which completes the proof of the implication (iii) $\Rightarrow$ (ii).

(ii) $\Rightarrow(\mathrm{i})$ : First, we can assume that $\varphi$ is real valued. By integration by parts we obtain that

$$
\left\|x^{\beta} \partial^{\alpha} \varphi(x)\right\|_{L^{2}}^{2}=\left|\int_{R^{n}} \partial^{\alpha}\left[x^{2 \beta} \partial^{\alpha} \varphi(x)\right] \varphi(x) d x\right| .
$$

Note that the boundary terms tend to zero by Theorem 1.2. Therefore, applying the Leibniz formula we have, for some constant $A$,

$$
\begin{aligned}
& \left\|x^{\beta} \partial^{\alpha} \varphi(x)\right\|_{L^{2}}^{2} \\
& \leqq \int_{R^{n}} \sum_{\substack{\gamma \leq \alpha \\
\gamma \leq 2 \beta}}\left(\begin{array}{c}
\alpha \\
\gamma
\end{array}\right)\left(\begin{array}{c}
2 \beta \\
\gamma
\end{array}\right) \gamma !\left|x^{2 \beta-\gamma} \partial^{2 \alpha-\gamma} \varphi(x)\right||\varphi(x)| d x \\
& \leqq n^{\prime \alpha \mid} \int_{\gamma \leq \alpha}\left(\begin{array}{c}
2 \beta \\
\gamma
\end{array}\right) \gamma !(2 \beta-\gamma) ! A^{|2 \beta-\gamma|}|\varphi(x)| \exp k|x| i \partial^{2 n-\gamma} \varphi(x) \mid \exp (-k|x| / 2) d x \\
& \leqq C_{1} n^{|a|} \sum_{\substack{\gamma \leq \alpha \\
\gamma \leq 2 \beta}}\left(\begin{array}{c}
2 \beta \\
\gamma
\end{array}\right)(2 \beta) ! A^{|2 \beta-\gamma|} M C h^{|2 \alpha-\gamma|}(2 \alpha-\gamma) !
\end{aligned}
$$

where $C_{1}=\int_{R n} \exp (-k|x| / 2)$ and $M \geqq \sup _{x}|\varphi(x)| \exp k|x|$. Here we use the inequality

$$
x^{a} \leqq \alpha ! \exp |x|
$$

for any $\alpha \in \boldsymbol{N}_{0}^{n}$. If we choose positive constants $A, h>1$ if necessary, and use the inequalities

$$
(\alpha !)^{2} \leqq(2 \alpha) ! \leqq n^{2 \mid \alpha_{1}}(\alpha !)^{2}
$$

we have, for some $C_{2}$

$$
\begin{aligned}
\left\|x^{\beta} \partial^{\alpha} \varphi\right\|_{L^{2}}^{2} & \leqq C_{2} n^{|\alpha|+2|\beta|}(2 \alpha) !(2 \beta) ! A^{2|\beta|} h^{2|a|} \\
& \leqq C_{2}(n A)^{4|\beta|}(n \sqrt{n} h)^{2|\alpha|}(\alpha !)^{2}(\beta !)^{2} .
\end{aligned}
$$

Thus we obtain that for some positive constants $C_{0}, C_{1}$ and $C_{2}$ such that

$$
\frac{\left\|x^{\beta} \partial^{\alpha} \varphi(x)\right\|_{L^{2}}}{C_{2}^{|\beta|} \beta !} \leqq C_{0} C_{1}^{|a|} \alpha !
$$

Therefore, summing up with respect to $\beta$ we can choose a positive constant 
$k$ such that

$$
\left\|\partial^{\alpha} \varphi(x) \exp k ! x \mid\right\|_{L^{2}} \leqq C_{0} C_{1}^{|\alpha|} \alpha ! .
$$

By the Cauchy-Schwarz inequality there exists a positive constant $C_{3}$ such that

$$
\begin{aligned}
\left\|\partial^{a} \varphi(x) \exp \frac{k}{2}|x|\right\|_{L^{1}} & \leqq\left\|\partial^{\alpha} \varphi(x) \exp k|x|\right\|_{L^{2}}\left[\int \exp (-k|x|) d x\right]^{1 / 2} \\
& \leqq C_{3} C_{1}^{|\alpha|} \alpha ! .
\end{aligned}
$$

Also, there exist positive constants $k$ and $C_{1}$ such that

$$
\left\|\partial^{\alpha} \varphi(x) \exp k \sqrt{1+|x|^{2}}\right\|_{L_{1}} \leqq C_{0} C_{1}^{|\alpha|} \alpha ! .
$$

Hence

$$
\begin{aligned}
& \left|\partial^{\alpha} \varphi(x) \exp k \sqrt{1+|x|^{2}}\right| \\
& =\left|\int_{-\infty}^{x_{n}} \cdots \int_{-\infty}^{x_{1}} \partial_{1} \cdots \partial_{n}\left(\partial^{\alpha} \varphi(x) \exp k \sqrt{1+|x|^{2}}\right) d x\right| \\
& \quad=\mid \int_{-\infty}^{x_{n}} \cdots \int_{-\infty}^{x_{1}} \partial_{n} \cdots \partial_{2}\left[\left(\partial_{1} \partial^{\alpha} \varphi\right) \exp k \sqrt{1+|x|^{2}}\right. \\
& \left.\quad+\partial^{\alpha} \varphi(x) \cdot \partial_{1}\left(\exp k \sqrt{1+|x|^{2}}\right)\right] d x \mid \\
& \quad \leqq \int_{-\infty}^{x_{n}} \cdots \int_{-\infty}^{x_{1}} \Sigma\left|\partial_{\jmath_{1}} \cdots \partial_{j_{r}}\left(\partial^{\alpha} \varphi\right)\right|\left|\left(\partial_{\jmath_{r+1}} \cdots \partial_{j_{n}}\right) \exp k \sqrt{1+|x|^{2}}\right| d x
\end{aligned}
$$

where the summation is taken over all $r=0,1, \cdots, n$ and $\left\{j_{1}, \cdots, j_{n}\right\}$ is a permutation of $\{1, \cdots, n\}$. We can prove by induction

$$
\left|\left(\partial_{\jmath_{1}} \cdots \partial_{j_{r}}\right) \exp k \sqrt{1+|x|^{2}}\right| \leqq P_{r}(k) \exp k \sqrt{1+|x|^{2}}
$$

where $P_{r}(k)$ is a polynomial of $k$ of $r$-th degree. Hence we derive that

$$
\begin{aligned}
& \left|\partial^{\alpha} \varphi(x) \exp k \sqrt{1+|x|^{2}}\right| \\
& \quad \leqq \int C \sum\left|P_{n-r}(k)\right|\left|\left(\partial_{j_{1}} \cdots \partial_{j_{r}}\right)\left(\partial^{\alpha} \varphi\right)\right| \exp k \sqrt{1+|x|^{2}} d x \\
& \quad \leqq C \sum\left|P_{n-r}(k)\right| C_{0} C_{1}^{|\alpha|+r}(\alpha+\beta) ! \\
& \quad \leqq C(k, n) C_{1}^{|\alpha|} \alpha !
\end{aligned}
$$

where $\beta$ is a multi-index with $|\beta|=r$. Therefore, using the relation

we obtain

$$
\exp k|x| \leqq \exp k \sqrt{1+|x|^{2}} \leqq e^{k} \exp k|x|
$$

$$
\sup _{x}\left|\partial^{\alpha} \varphi(x)\right| \exp k|x| \leqq C(k, n) C_{1}^{|\alpha|} \alpha !
$$

which completes the proof.

Now we can rephrase Theorem 2.1 as follows. 
Theorem 2.2. The space $F$ of test functions for the Fourier hyperfunctions consists of all locally integrable functions such that for some $h, k>0$

$$
\begin{aligned}
& \sup _{x}|\varphi(x)| \exp k|x|<\infty, \\
& \sup _{\xi}|\hat{\varphi}(\xi)| \exp h|\xi|<\infty .
\end{aligned}
$$

Remark. Combining Theorem 1.2 on the Schwartz space $\mathcal{S}$ and Theorem 2.2 on the space $\mathscr{I}$ we can easily compare the spaces $\mathcal{S}$ and $\mathscr{I}$ which are both invariant under the Fourier transformations as follows:

(i) The space $\mathcal{S}$ consists of all $C^{\infty}$ functions $\varphi$ such that $\varphi$ itself and its Fourier transform $\hat{\varphi}$ are both rapidly decreasing.

(ii) The space $\mathscr{T}$ consists of all $C^{\infty}$ functions $\varphi$ such that $\varphi$ itself and its Fourier transform $\hat{\varphi}$ are both exponentially decreasing.

\section{Acknowledgement}

The authors are grateful to Professor A. Kaneko for suggesting the problem and to the referee for the helpful suggestions.

\section{References}

[1] Chung, J., Chung, S.Y. and Kim, D., Une caractérisation d'espace de Schwartz, C.R. Acad. Sci. Paris Sér. I Math., 316 (1993), 23-25.

[2] Hörmander, L., The analysis of linear partial differential operator I, SpringerVerlag, Berlin-New York, 1983.

[3] — Between distributions and hyperfunctions, Astérisque, 131 (1985), 89106.

[4] Kaneko, A., Introduction to hyperfunctions, KTK Scientific Publishers, Tokyo, 1988.

[5] Kawai, T., On the theory of Fourier hyperfunctions and its applications to partial differential equations with constant coefficients, J. Fac. Sci. Univ. Tokyo, Sect. IA, 17 (1970), 467-517.

[6] Kim, K.W., Chung, S.Y. and Kim, D., Fourier hyperfunctions as the boundary values of smooth solutions of the heat equation, Publ. RIMS, Kyoto Univ., 29 (1993), 287-300. 